\title{
ADRC Attitude Controller Design for Hypersonic Vehicle based on MIMO- ESO
}

\author{
JianHua Wen ${ }^{1}$, ChangMao Qin ${ }^{1}$ and Xin Zhang ${ }^{1}$ \\ ${ }^{1}$ Senior Engineer, China Academy of Launch Vehicle Technology,Mailbox 9200 sub box 1-1,Beijing, 100076, China
}

\begin{abstract}
For the hypersonic vehicle nonlinear attitude mode in reentry process with a strong coupling, aerodynamic parameter perturbations and non-deterministic, combine extended state observer and nonlinear law state error feedback, design the hypersonic vehicle MIMO-ESO ADRC attitude controller. Put interference such as uncertainty, coupling and parameter perturbations as "the sum of interference" ,use the extended state observer to estimate and dynamic feedback compensation, use nonlinear law state error feedback to inhibit residual of compensation. ADRC controller is charged without a precise model of vehicle, and without precise perturbation boundaries of aerodynamic parameters.Simulation results show that the MIMO-ESO ADRC attitude controller can overcome the impact of largescale perturbations of interference and aerodynamic parameters, have good dynamic qualities and tracking capabilities, also have strong robustness.
\end{abstract}

\section{Introduction}

Hypersonic vehicle has important military significance. It has become a research hotspot in various countries in recent years ${ }^{[1]}$. The controller design has been more and more difficult due to the complex nonlinearity, the strong coupling between control channels and the uncertainties of aerodynamic parameters in the non-power re-entry process. In existing methods such as robust control ${ }^{[2,3]}$ and adaptive control ${ }^{[4,5]}$, the linearized model of the controlled system contains complex high-order derivative function, which is not convenient for practical application of engineering. Sliding mode control ${ }^{[6,7]}$ compensates the influence of uncertainty by designing the virtual control quantity, but the sliding mode control is based on the upper bound of uncertainty and requires that its bound is known or the known function of the state variables, which is difficult to predict in practical applications.

The active disturbance rejection controller (ADRC) ${ }^{[8]}$ is not dependent on the system model, but is a method that relies on the process error to eliminate the error. The extended state observer (ESO) estimates the "summation perturbation" to obtain the real-time effect amount of object model's internal and external disturbances, and with the real-time dynamic feedback compensation system is successfully linearized. In the meantime, nonlinear feedback control law (NLSEF) are used to suppress compensation residuals and improve control performance $^{[9]}$. After more than ten years of development, ADRC technology has gradually matured. In 2013, Texas Instruments released a global motion control chip with $\mathrm{ADRC}$ as the core, further demonstrating the great potential of ADRC technology in engineering applications ${ }^{[10,11]}$.

In this paper, the MIMO-ESO auto disturbancerejection attitude controller is designed based on the nonlinear attitude model of the hypersonic vehicle's nonpower re-entry. Uncertain items, unmodeled dynamics, coupling effects, parameter perturbations, and interference influences are considered as "summation interferences." ESO are used for estimation and dynamic feedback compensation, and NLSEF is used to suppress the compensation residuals. Depending on the characteristics that the auto-disturbance rejection is not relying on the model, the problem of complex linearization model and sliding model control requires perturbation is successfully solved. Selecting parameters according to ESO stability conditions can obtain good dynamic quality and tracking performance, and can also overcome the influence of interference and aerodynamic parameters over a wide range of perturbations, and has strong robustness.

\section{Hypersonic Vehicle Modeling}

The non-power re-entry attitude model of a hypersonic vehicle is a nonlinear system with uncertain items as follows:

$$
\dot{\boldsymbol{x}}=f(x)+g(x) \delta
$$

As $x=\left[\begin{array}{llllll}\alpha & \beta & \gamma_{c} & \omega_{x} & \omega_{y} & \omega_{z}\end{array}\right]^{T}, \delta=\left[\begin{array}{lll}\delta_{e} & \delta_{a} & \delta_{r}\end{array}\right]^{T}$.

Among them:

$$
f(x)=\left[\begin{array}{llllll}
f_{\alpha} & f_{\beta} & f_{\gamma_{c}} & f_{\omega_{x}} & f_{\omega_{y}} & f_{\omega_{z}}
\end{array}\right]^{T}
$$


$f_{\alpha}=\omega_{z}-\omega_{x} \cos \alpha \tan \beta+\omega_{y} \sin \alpha \tan \beta$

$-\frac{1}{m V \cos \beta}\left[q S C_{Y, \alpha}+m g \cos \theta \cos \gamma_{c}\right]$

$f_{\beta}=\omega_{x} \sin \alpha+\omega_{y} \cos \alpha$

$+\frac{1}{m V}\left[q S C_{Z, \beta} \beta-m g \cos \theta \sin \gamma_{c}\right]$

$f_{\gamma_{c}}=\omega_{x} \frac{\cos \alpha}{\cos \beta}-\omega_{y} \frac{\sin \alpha}{\cos \beta}+\frac{g}{V} \cos \theta \cos \gamma_{c} \tan \beta$

$+\frac{1}{m V}\left[q S C_{Y, \alpha}\left(\sin \theta \sin \gamma_{c}+\tan \beta\right)+q S C_{Z, \beta} \beta \sin \theta \cos \gamma_{c}\right]$

$f_{\omega_{x}}=\frac{\left(I_{y}-I_{z}\right)}{I_{x}} \omega_{y} \omega_{z}+\frac{1}{I_{x}} q b S\left(C_{l, \beta} \beta\right.$

$\left.+C_{l, \omega_{x}} \frac{\omega_{x} b}{2 V}+C_{l, \omega_{y}} \frac{\omega_{y} b}{2 V}\right)$

$f_{\omega_{y}}=\frac{\left(I_{z}-I_{x}\right)}{I_{y}} \omega_{x} \omega_{z}+\frac{1}{I_{y}} q b S\left(C_{m, \beta} \beta\right.$

$\left.+C_{m, \omega_{x}} \frac{\omega_{x} b}{2 V}+C_{m, \omega_{y}} \frac{\omega_{y} b}{2 V}\right)+\frac{1}{I_{y}} X_{c g} q S C_{Z, \beta} \beta$

$f_{\omega_{z}}=\frac{\left(I_{x}-I_{y}\right)}{I_{z}} \omega_{y} \omega_{x}+\frac{1}{I_{z}} q c S\left(C_{n, \alpha}\right.$

$\left.+C_{n, \omega_{z}} \frac{\omega_{z} c}{2 V}\right)+\frac{1}{I_{z}} X_{c g} q S\left(C_{X, \alpha} \sin \alpha+C_{Y, \alpha} \cos \alpha\right)$

$g(x)=\left[\begin{array}{ccc}g_{\alpha, \delta_{e}} & g_{\alpha, \delta_{a}} & 0 \\ g_{\beta, \delta_{e}} & g_{\beta, \delta_{a}} & g_{\beta, \delta_{r}} \\ g_{\gamma_{c}, \delta_{e}} & g_{\gamma_{c}, \delta_{a}} & g_{\gamma_{c}, \delta_{r}} \\ g_{\omega_{x}, \delta_{e}} & g_{\omega_{x}, \delta_{a}} & g_{\omega_{x}, \delta_{r}} \\ g_{\omega_{y}, \delta_{e}} & g_{\omega_{y}, \delta_{a}} & g_{\omega_{y}, \delta_{r}} \\ g_{\omega_{z}, \delta_{e}} & g_{\omega_{z}, \delta_{a}} & g_{\omega_{z}, \delta_{r}}\end{array}\right]$

$g_{\alpha, \delta_{e}}=-\frac{q S C_{Y, \delta_{e}}}{m V \cos \beta}, g_{\alpha, \delta_{a}}=-\frac{q S C_{Y, \delta_{a}}}{m V \cos \beta}, g_{\beta, \delta_{e}}=\frac{q S C_{Z, \delta_{e}}}{m V}$

$g_{\beta, \delta_{a}}=\frac{q S C_{Z, \delta_{a}}}{m V}, g_{\beta, \delta_{r}}=\frac{q S C_{Z, \delta_{r}}}{m V}$

$g_{\gamma_{c}, \delta_{e}}=\frac{q S\left[C_{Y, \delta_{e}}\left(\sin \theta \sin \gamma_{c}+\tan \beta\right)+C_{Z, \delta_{e}} \sin \theta \cos \gamma_{c}\right]}{m V}$

$g_{\gamma_{c}, \delta_{a}}=\frac{q S\left[C_{Y, \delta_{a}}\left(\sin \theta \sin \gamma_{c}+\tan \beta\right)+C_{Z, \delta_{a}} \sin \theta \cos \gamma_{c}\right]}{m V}$

$g_{\gamma_{c}, \delta_{r}}=\frac{q S C_{Z, \delta_{r}} \sin \theta \cos \gamma_{c}}{m V}, g_{\omega_{x}, \delta_{e}}=\frac{q b S C_{l, \delta_{e}}}{I_{x}}$

$g_{\omega_{x}, \delta_{a}}=\frac{q b S C_{l, \delta_{a}}}{I_{x}}, g_{\omega_{x}, \delta_{r}}=\frac{q b S C_{l, \delta_{r}}}{I_{x}}$

$g_{\omega_{y}, \delta_{e}}=\frac{q b S C_{m, \delta_{e}}}{I_{y}}+\frac{X_{c g} q S C_{Z, \delta_{e}}}{I_{y}}$

$g_{\omega_{y}, \delta_{a}}=\frac{q b S C_{m, \delta_{a}}}{I_{y}}+\frac{X_{c g} q S C_{Z, \delta_{a}}}{I_{y}}$

$$
\begin{aligned}
& g_{\omega_{y}, \delta_{r}}=\frac{q b S C_{m, \delta_{r}}}{I_{y}}+\frac{X_{c g} q S C_{Z, \delta_{r}}}{I_{y}} \\
& g_{\omega_{z}, \delta_{e}}=\frac{q c S C_{n, \delta_{e}}}{I_{z}}+\frac{X_{c g} q S\left(\sin \alpha C_{X, \delta_{e}}+\cos \alpha C_{Y, \delta_{e}}\right)}{I_{z}} \\
& g_{\omega_{z}, \delta_{a}}=\frac{q c S C_{n, \delta_{a}}}{I_{z}}+\frac{X_{c g} q S\left(\sin \alpha C_{X, \delta_{a}}+\cos \alpha C_{Y, \delta_{a}}\right)}{I_{z}} \\
& g_{\omega_{z}, \delta_{r}}=\frac{q c S C_{n, \delta_{r}}}{I_{z}}+\frac{X_{c g} q S \sin \alpha C_{X, \delta_{r}}}{I_{z}}
\end{aligned}
$$

Among the above symbols, $g_{i, j}$ is an aerodynamic parameter item. Its specific parameters are described in reference [12]. Different from ordinary low-speed vehicles, each aerodynamic parameter term is a function of angle of attack and Mach number, and contains uncertainties caused by perturbation of aerodynamic parameters, which enhances the coupling between channels and leads to more complex and difficult controller design.

\section{Auto-Disturbance Rejection Control Technology Based on MIMO-ESO}

\subsection{MIMO-ESO ADRC design}

For system (1), the design of the extended state observer ESO is the core of the entire auto-disturbance rejection controller. Each channel is a first-order system, so only the second-order extended state observer ESO needs to be reconstructed. The input matrix parameter $B$ of the control variable $\delta$ is related to the aerodynamic parameter. Although there are related parameters that can be referenced, it is not an exact value, so $B$ takes the reference aerodynamic parameter as the nominal value $B_{0}$. Since $f(\boldsymbol{x})$ also has parameter perturbations and uncertainties, it is replaced by $f(\beth) . f(\beth)$ is the total uncertainty, including $f(\boldsymbol{x})$ and external environmental disturbances, unmodeled dynamics, coupling effects, and other disturbances.

Replace $g(x)$ with the estimated value $g_{0}, f(\beth)$ instead of $f(\boldsymbol{x})$, the system (1) can be equivalent to:

$$
\dot{x}=a(t)+U
$$

Among them:

$$
\begin{gathered}
a(t)=f(\square)+\left(g(x)-g_{0}\right) \delta \\
U=g_{0} \delta
\end{gathered}
$$

The system measurement equation is:

$$
y=x=\left[\begin{array}{llllll}
\alpha & \beta & \gamma_{c} & \omega_{x} & \omega_{y} & \omega_{z}
\end{array}\right]^{T}
$$

The second-order ESO with the same structure and the same parameters is configured for the six channels of the system (2). The generalized second-order MIMO-ESO equation is:

$$
\begin{aligned}
& \mathbf{e}_{1}=\mathbf{z}_{11}-x \\
& \dot{\mathbf{z}}_{11}=\mathbf{z}_{12}-b_{11} \mathbf{e}_{1}+U \\
& \dot{\mathbf{z}}_{12}=-b_{12} \mathbf{f a l}\left(\mathbf{e}_{1}, a_{1}, \sigma_{1}\right)
\end{aligned}
$$

Among them $b_{11}>0, b_{12}>0,0<a_{1}<1, \sigma_{1}$ are all 
scalar. $\mathbf{e}_{1}, \mathbf{z}_{11}, \dot{\mathbf{z}}_{11}, \mathbf{z}_{12}, \dot{\mathbf{z}}_{12}$ are all $6 \times 1$ vectors.

$$
\mathbf{e}_{1}=\left[\begin{array}{l}
e_{11} \\
e_{12} \\
e_{13} \\
e_{14} \\
e_{15} \\
e_{16}
\end{array}\right], \mathbf{f a l}\left(\mathbf{e}_{1}, a_{1}, \sigma_{1}\right)=\left[\begin{array}{c}
\operatorname{fal}\left(e_{11}, a_{1}, \sigma_{1}\right) \\
\operatorname{fal}\left(e_{12}, a_{1}, \sigma_{1}\right) \\
\operatorname{fal}\left(e_{13}, a_{1}, \sigma_{1}\right) \\
\operatorname{fal}\left(e_{14}, a_{1}, \sigma_{1}\right) \\
\operatorname{fal}\left(e_{15}, a_{1}, \sigma_{1}\right) \\
\operatorname{fal}\left(e_{16}, a_{1}, \sigma_{1}\right)
\end{array}\right]
$$

The nonlinear function $\operatorname{fal}(e, a, \sigma)$ in the above formula is:

$$
f a l(e, a, \delta)=\left\{\begin{array}{cc}
e \times \delta^{a-1} & |e| \leq \delta \\
|e|^{a} \operatorname{sgn}(e) & |e|>\delta
\end{array}\right.
$$

Among them $\operatorname{sgn}(e)$ is a symbolic function of $\mathrm{e}$.

As long as the parameter selection in the ESO is appropriate and satisfies the following second-order ESO stability conditions: $b_{11}^{2}>4 b_{12} \sigma_{1}^{a_{1}-1}$, then in the steady state, the ESO state will have the following convergence relationship:

$$
\mathbf{z}_{11} \rightarrow x, \mathbf{z}_{12} \rightarrow a(t)=f(\square)+\left(g(x)-g_{0}\right) \delta
$$

The ESO status will estimate the projectile angular velocity and the total uncertainties $a(t)$ in the model in real time. Using the estimated values $\mathbf{z}_{12}$, implement the following dynamic feedback compensation laws for system (2):

$$
U=U_{0}-\mathbf{z}_{12}
$$

The system (2) is then dynamically linearized into a single-integral system: $\dot{\boldsymbol{x}}=U_{0}$.

It can be seen that after dynamic compensation, the six channels from control input $U_{0}$ to output $x$ become parallel six single integrator systems, six channels are decoupled, and this dynamic feedback compensation is implemented using ESO without known $f(\beth)$ and accurate parameter values. This method allows parametric perturbations, uncertainties, and interference effects, eliminating the need for accurate dynamic modelling of the body's attitude.

In order to quickly suppress the compensation residual, the control law uses a nonlinear state error feedback law (NLSEF) with a nonlinear (non-smooth) feedback effect to control the integral system.

$$
\begin{aligned}
& \mathbf{e}_{0}=\boldsymbol{x}^{*}-\mathbf{z}_{11} \\
& U_{0}=b_{0} \mathbf{f a l}\left(\mathbf{e}_{0}, a_{0}, \sigma_{0}\right)
\end{aligned}
$$

Among them, $\mathbf{e}_{0}=\left[\begin{array}{llllll}e_{01} & e_{02} & e_{03} & e_{04} & e_{05} & e_{06}\end{array}\right]^{T}$, $x^{*}=\left[\begin{array}{llllll}\alpha^{*} & \beta^{*} & \gamma_{c}^{*} & \omega_{x}^{*} & \omega_{y}^{*} & \omega_{z}^{*}\end{array}\right]^{T}$

In the above formula, $b_{0}>0,0<a_{0}<1, \sigma_{0}$ can be adjusted to avoid flutter near the zero point. $\left[\alpha^{*}, \beta^{*}, \gamma_{c}^{*}\right]$ is the desired attitude angle, and $\left[\omega_{x}^{*}, \omega_{y}^{*}, \omega_{z}^{*}\right]$ is the desired angular velocity needed to track the desired attitude angle, which is the real-time simulation quantity.

From equation (2), the control command for the vehicle's steering surface $\delta$ can be obtained (the following formula is called static decoupling law):

$$
\delta=g_{0}^{-1} U
$$

Equations (3) to (6) constitute an auto-disturbancedistorting attitude controller.

\subsection{Stability Analysis and Error Analysis}

System (1) becomes the following system under the action of the MIMO-ESO auto disturbance rejection controller:

Among them:

$$
\begin{aligned}
& \dot{\boldsymbol{x}}=a(t)+U_{0}-\mathbf{z}_{12} \\
& U_{0}=b_{0} \mathbf{f a l}\left(\mathbf{e}_{0}, a_{0}, \sigma_{0}\right)
\end{aligned}
$$

$$
\begin{gathered}
\mathbf{e}_{1}=\mathbf{z}_{11}-x, \mathbf{e}_{2}=\mathbf{z}_{12}-\mathbf{a}(\mathbf{t}) \\
\dot{\mathbf{e}}_{1}=\mathbf{e}_{2}-b_{11} \mathbf{e}_{1}, \dot{\mathbf{e}}_{2}=-b_{12} \mathbf{f a l}\left(\mathbf{e}_{1}, a_{1}, \sigma_{1}\right)-\dot{a}(\mathbf{t})
\end{gathered}
$$

The following demonstrates the stability of the autodisturbance rejection control system. First define the following function:

$$
\begin{gathered}
V_{1}=\frac{1}{2} \sum_{i=1}^{m} g_{2 \mathrm{i}}^{2}\left(e_{1 \mathrm{i}}, e_{2 \mathrm{i}}\right) \\
g_{2 i}\left(e_{1 i}, e_{2 i}\right)=\left\{\begin{array}{c}
\left|h_{2 i}\left(e_{1 i}, e_{2 i}\right)\right|\left|h_{2 i}\left(e_{1 i}, e_{2 i}\right)\right|>g_{1 i}\left(e_{1 i}\right) \\
g_{1 i}\left(e_{1 i}\right) \quad\left|h_{2 i}\left(e_{1 i}, e_{2 i}\right)\right| \leq g_{1 i}\left(e_{1 i}\right)
\end{array}\right. \\
h_{2 i}\left(e_{1 i}, e_{2 i}\right)=e_{2 i}-b_{11} e_{1 i}+k g_{1 i}\left(e_{1 i}\right) \operatorname{sgn}\left(e_{1 i}\right) \\
g_{1 i}\left(e_{1 i}\right)=\frac{b_{12}}{k b_{11}}\left|\operatorname{fal}\left(e_{1 i}, a_{1}, \sigma_{1}\right)\right|, \mathrm{k}>1, \mathrm{i}=1, \cdots, \mathrm{m}
\end{gathered}
$$

Lemma $1^{[13]}: G_{2}=\left\{\left(e_{1 i}, e_{2 i}\right):\left|h_{2 i}\left(e_{1 i}, e_{2 i}\right)\right| \leq g_{1 i}\left(e_{1 i}\right)\right\}$ is the self-stabilizing domain of the system.

Theorem 1: For the system (7), suppose that the expansion state quantity $a(t)$ is bounded by the time variation $-\dot{a}(t)=\left[-\dot{a}_{1}(t), \ldots,-\dot{a}_{m}(t)\right]^{T}, \quad$ ie, $\quad$ if $\left|-\dot{a}_{i}(t)\right|<w, i=1,2, \cdots, m$ then $w>0$, if the system satisfies:

$$
\begin{gathered}
b_{11}^{2}>(1+k) c_{2} b_{12} \sigma^{a_{1}-1} \\
b_{11} g_{2 i}>\frac{c_{2}}{c_{2}-1} w\left(c_{2}>1, i=1, \cdots, m\right)
\end{gathered}
$$

Then $\dot{V}_{1}<0$, the system is a stable system.

Provement: $\dot{V}_{1}=\sum_{i=1}^{m}\left[\mathrm{~g}_{2 i}\left(\mathrm{e}_{1 i}, \mathrm{e}_{2 i}\right) \dot{g}_{2 i}\left(\mathrm{e}_{1 \mathrm{i}}, \mathrm{e}_{2 \mathrm{i}}\right)\right]$,

When $\left|h_{2 i}\left(e_{1 i}, e_{2 i}\right)\right|>g_{1 i}\left(e_{1 i}\right)$, then,

$$
\begin{aligned}
& \dot{V}_{1 i}=g_{2 i}\left(\mathrm{e}_{1 \mathrm{i}}, \mathrm{e}_{2 \mathrm{i}}\right) \dot{g}_{2 i}\left(\mathrm{e}_{1 i}, \mathrm{e}_{2 i}\right)=h_{2 i} \dot{h}_{2 i} \\
& =h_{2 i}\left[\frac{\partial h_{2 i}}{\partial e_{2 i}} \cdot \dot{e}_{2 i}+\frac{\partial h_{2 i}}{\partial e_{1 i}} \cdot \dot{e}_{1 i}\right] \\
& <h_{2 i} w+\frac{\partial h_{2 i}}{\partial e_{1 i}} h_{2 i}^{2}-h_{2 i}\left(b_{12} \frac{\left|f a l_{1 i}\right|}{g_{1 i}}+k \frac{\partial h_{2 i}}{\partial e_{1 i}}\right) g_{1 i} \operatorname{sgn}\left(\mathrm{e}_{1 i}\right) \\
& =h_{2 i} w+\frac{\partial h_{2 i}}{\partial e_{1 i}} h_{2 i}^{2}+k^{2} h_{2 i} g_{1 i}\left|\frac{d g_{1 i}}{d e_{1 i}}\right| \\
& \leq h_{2 i} w-b_{11} h_{2 i}^{2}+k h_{2 i}^{2}\left|\frac{d g_{1 i}}{d e_{1 i}}\right|+k^{2} h_{2 i} g_{1 i}\left|\frac{d g_{1 i}}{d e_{1 i}}\right|
\end{aligned}
$$

Substituting equation (12a)、(12b) for availability: 


$$
\begin{aligned}
& \dot{V}_{1 i} \leq \frac{\left(c_{2}-1\right)}{c_{2}} b_{11} h_{2 i} g_{1 i}-b_{11} h_{2 i}^{2}+(1+k) h_{2 i}^{2} \frac{b_{12}}{b_{11}}\left|\frac{d f a l_{1 i}}{d e_{1 i}}\right| \\
& =-\frac{1}{c_{2}} b_{11} h_{2 i}^{2}+(1+k) h_{2 i}^{2} \frac{b_{12}}{b_{11}}\left|\frac{d f a l_{1 i}}{d e_{1 i}}\right|<0
\end{aligned}
$$

Established by the above formula, can be set:

$$
b_{11}^{2}>(1+k) c_{2} b_{12}\left|\frac{d f a l_{1 i}}{d e_{1 i}}\right|
$$

If $d f a l_{1 i}=\left|e_{1 i}\right|^{a_{1}}$, set $b_{11}^{2}>(1+k) c_{2} b_{12} \mathrm{a}_{1}\left|e_{1 i}\right|^{a_{1}-1}$,

When $e_{1 i} \rightarrow 0$,then $b_{11} \rightarrow \infty$.

To substitute $f a l_{1 i}=\left\{\begin{array}{cc}\left|e_{1 i}\right|^{a_{1}} \operatorname{sgn}\left(e_{1 i}\right) & \left|e_{1 i}\right|>\sigma \\ e_{1} \sigma^{a_{1}-1} & \left|e_{1 i}\right| \leq \sigma\end{array}\right.$ for this, you can get $b_{11}^{2}>(1+k) c_{2} b_{12} \sigma^{a_{1}-1}$, when formula (13) is satisfied, $\dot{V}_{1 i}<0$ and $\dot{V}_{1}=\sum_{1}^{m} \dot{V}_{1 i}<0$.

When $\left|h_{2 i}\left(e_{1 i}, e_{2 i}\right)\right| \leq g_{1 i}\left(e_{1 i}\right)$, then,

$$
\begin{aligned}
& \left|e_{2 i}-b_{11} e_{1 i}+k g_{1 i} \operatorname{sgn}\left(e_{1 i}\right)\right| \leq g_{1 i} \\
& -k g_{1 i} \operatorname{sgn}\left(e_{1 i}\right)-g_{1 i} \leq e_{2 i}-b_{11} e_{1 i} \leq-k g_{1 i} \operatorname{sgn}\left(e_{1 i}\right)+g_{1 i}
\end{aligned}
$$$$
\dot{V}_{1 i}=\left(\frac{b_{12}}{k b_{11}}\right)^{2}\left|f a l_{1 i}\right|\left|\frac{d f a l_{1 i}}{d e_{1 i}}\right| \dot{e}_{1 i} \operatorname{sgn}\left(e_{1 i}\right)
$$$$
=\left(\frac{b_{12}}{k b_{11}}\right)^{2}\left|f a l_{1 i}\right|\left|\frac{d f a l_{1 i}}{d e_{1 i}}\right| \frac{e_{1 i} \dot{e}_{1 i}}{\left|e_{1 i}\right|}
$$

$$
\begin{aligned}
& \text { Set }\left(\frac{b_{12}}{k b_{11}}\right)^{2}\left|f a l_{1 i}\right|\left|\frac{d f a l_{1 i}}{d e_{1 i}}\right|=F, \text { then, } \\
& \dot{V}_{1 i}=F e_{1 i} \dot{e}_{1 i}=F e_{1 i}\left(e_{2 i}-b_{11} e_{1 i}\right) \leq F e_{1 i}\left(-k g_{1 i} \operatorname{sgn}\left(e_{1 i}\right)+g_{1 i}\right) \\
& =F\left(-k g_{1 i}\left|e_{1 i}\right|+g_{1 i} e_{1 i}\right) \\
& \quad<-F(k-1) g_{1 i}\left|e_{1 i}\right|<0
\end{aligned}
$$

Therefore, Theorem 1 proves.

According to the theorems (1) and (12b), $g_{1 i}=\frac{c_{2}}{\left(c_{2}-1\right) b_{11}} w$ is the boundary of the self-stability domain.

$$
\begin{cases}\left|h_{2 i}\right|=\frac{c_{2}}{\left(c_{2}-1\right) b_{11}} w & \left|h_{2 i}\right|>g_{1 i} \\ g_{1 i}=\frac{c_{2}}{\left(c_{2}-1\right) b_{11}} w & \left|h_{2 i}\right| \leq g_{1 i}\end{cases}
$$

As

$$
\begin{gathered}
e_{1}^{*}=\max \left\{\left|e_{1 i}\right|\right\}=\left[\frac{k c_{2} w}{b_{12}\left(c_{2}-1\right)}\right]^{\frac{1}{a_{1}}} \\
e_{2}^{*}=\max \left\{\left|e_{2 i}\right|\right\}=b_{11} \mathrm{e}_{1}^{*}-\frac{(k-1) c_{2} w}{b_{11}\left(c_{2}-1\right)}
\end{gathered}
$$

In the formula, $\mathrm{e}_{1}^{*}$ and $\mathrm{e}_{2}^{*}$ are the maximum estimation errors of the response expansion observer to the state quantity $x(t)$ and the expansion state quantity $a(t)$, respectively.
When $b_{11}>\frac{k c_{2} w}{\left(c_{2}-1\right)}, a_{1}$ is smaller, $\mathrm{e}_{1}^{*}$ and $\mathrm{e}_{2}^{*}$ are smaller too. Which means the smaller $\mathrm{B}$ is, the more accurate the estimation of the disturbance of the extended observer on the sum is, and the stronger the antiinterference ability is.

Redefine the function $V_{2}=\frac{1}{2}\left(x^{*}-x\right)^{T}\left(x^{*}-x\right)$,

$$
\begin{aligned}
\dot{V}_{2} & =\frac{1}{2}\left(\dot{x}^{*}-\dot{x}\right)^{T}\left(x^{*}-x\right)=\left(\dot{x}^{*}-a(t)-U_{0}+z_{12}\right)^{T}\left(x^{*}-x\right) \\
& =-V_{0}^{T}\left(x^{*}-x\right)+\left(\dot{x}^{*}-a(t)+z_{12}\right)^{T}\left(x^{*}-x\right) \\
& =-b_{0} \sum_{i=1}^{m}\left|\left(x^{*}-x\right)\right|^{1=a_{0}}+\left(\dot{x}^{*}-e_{2}\right)^{T}\left(x^{*}-x\right)
\end{aligned}
$$

Thus, when the following inequality is satisfied:

$$
b_{0}\left|\left(x^{*}-x\right)_{i}\right|>\left|\left[\dot{x}^{*}+e_{2}\right]_{i}\right|
$$

$\dot{V}_{2}<0$ is Established.

Among them, $[A]_{i}$ is the ith element of $\mathrm{A}$.

Therefore, the static tracking error of the selfinterference control system is

$$
\left|\left(x^{*}-x\right)_{i}\right|<\left|\frac{\left[\dot{x}^{*}+e_{2}\right]_{i}}{b_{0}}\right|^{\frac{1}{a_{0}}}
$$

When $b_{0}>\left|\left[\dot{x}^{*}+e_{2}\right]_{i}\right|$, the smaller $a_{0}$ is, the smaller the tracking error is.

Definition $V=V_{1}+V_{2}$, when satisfying both (12) and (15), $\dot{V}=\dot{V}_{1}+\dot{V}_{2}<0$ the self-interference control system is stable.

\section{Simulation analysis}

A certain type of BTT vehicle model is used as an example for simulation. $\alpha^{*}$ and $\gamma_{c}^{*}$ are shown in the guidance command signals corresponding to the solid lines in Fig. 1 and Fig. 3, respectively. The coordination requirement of the BTT vehicle directly leads to $\beta^{*}=0$.

Select the desired input $\left[\alpha^{*}, \beta^{*}, \gamma_{c}^{*}\right]=[5,0,5]$. The range of the perturbation of the aerodynamic parameters in the simulation is $\pm 50 \%$. Assess the performance of the control system in the case of a wide range of aerodynamic parameters.

Select design parameters: $b_{11}=80, b_{12}=0.001$, $a_{1}=0.5, \sigma_{1}=0.01 ; b_{0}=200, a_{0}=1, \sigma_{0}=0.01$.

The simulation results are shown in Figure $1 \sim$ Figure 3. Figure 1 shows the attack angle response curve, Figure 2 shows the slip angle response curve, and Figure 3 shows the tilt angle response curve. Among them, the solid line is the tracking curve under the standard parameters, the broken line is the tracking curve when the aerodynamic parameter is perturbed by $50 \%$, and the dotted line is the tracking curve when the aerodynamic parameter is perturbed by $-50 \%$. 


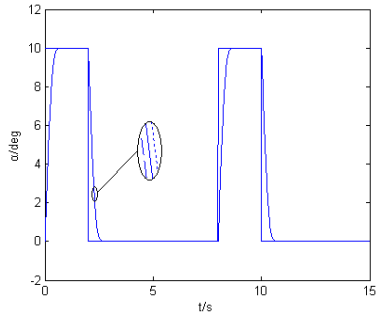

Figure 1. Attack angle curve

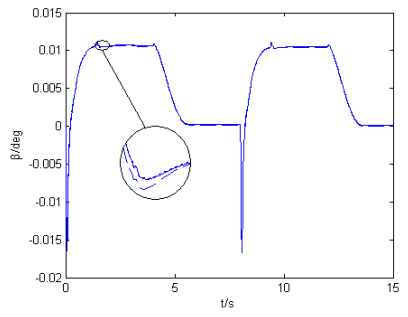

Figure 2. slip angle curve

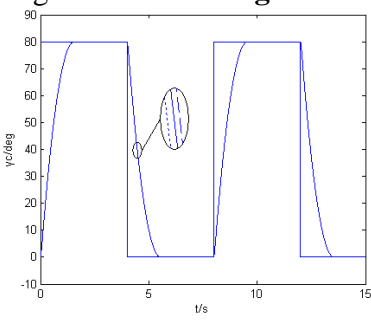

Figure 3. Tilt angle curve

The simulation results show that in the case of standard parameters, both the angle of attack and the angle of roll can track guidance command signal quickly and without overshooting, with good dynamic quality and high tracking accuracy, and the side slip angle satisfies the index $|\beta| \leq 0.3^{\circ}$. The maximum rudder deflection angle of the vehicle is 10 degrees, and the maximum rudder deflection speed is 40 degrees/s. In the case of large perturbation of aerodynamic parameters, the three channels can also meet the stability requirements, and the control system still exhibits good stability and tracking performance, and has strong robustness.

\section{Conclusion}

In this paper, the MIMO-ESO auto disturbancerejection attitude controller for hypersonic vehicle is designed based on the nonlinear attitude model for hypersonic vehicles' re-entry and the extended state observer and nonlinear state error feedback law in auto disturbance rejection control. Simulations show that the control system can overcome the influence of disturbance and aerodynamic parameters over a wide range of perturbations, and it has strong robustness while obtaining good dynamic quality and tracking performance. Because the extended state observer estimates the uncertainties, unmodeled dynamics, coupling effects, parametric perturbations, and interference effects as "summation interference" and dynamically compensates for feedback, the nonlinear residual state error feedback law is used to suppress the compensation residuals. The design of the ADRC is not required to accurately control the model of the vehicle, and only a standard value or an estimated value is required for the aerodynamic parameters. It is not necessary to know the perturbation limits of the aerodynamic parameters either. It successfully overcomes the difficulties in establishing an accurate controlled model and obtaining the parameter perturbation range in actual projects. Therefore, it has great engineering application value.

\section{References}

1. BOLENDER M A. An Overview on Dynamics and Controls Modelling of Hypersonic Vehicles. 2009 American Control Conference,pp.2507-2512.

2. WANG Q,STENGEL R F.Robust nonlinear control of a hypersonic aircraft.Journal of Guidance, Control and Dynamics, 2000,23(4),pp.577-584.

3. ITO D,WARD D,VALASEK J. Robust dynamic inversion controller design and analysis for the X-38. AIAA Guidance,Navigation, and Control Conference. Montreal, Canada,2001.

4. XU H J,MIRMIRANI M,IOANNOU P A.Robust neural adaptive control of a hypersonic aircraft.AIAA Guidance, Navigation, and Control conference and Exhibit. Austin, Texas,2003.

5. WALLNER E M,WELL $\mathrm{K}$ H.Nonlinear flight control design for the X-38 using CMAC neural networks.AIAA Guidance, Navigation, and Control Conference and Exhibit. Montreal, Canada, 2001.

6. XU H J,IOANNOU P A,MIRMIRANI M.Adaptive sliding mode control design for a hypersonic flight vehicle. Journal of Guidance, Control and Dynamics, 2004,25(5),pp.829-838.

7. Junchun Yang, Jun Hu, Xiaole Lv.Design of Sliding Mode Tracking Control for Hypersonic Reentry Vehicles. Proceedings of the 26th Chinese Control Conference, Zhangjiajie, Hunan, China, 2007.

8. Han J. From PID to active disturbance rejection control. Industrial Electronics IEEE transactions on, 2009, 56(3) ,pp.900-906.

9. Qi N M, Qin C M, Song Z G. Improved ADRC cascade decoupling controller design of hypersonic vehicle. Harbin Gongye Daxue Xuebao(Journal of Harbin Institute of Technology), 2011, 43(11),pp.3438.

10. Herbst G. A Simulative Study on Active Disturbance Rejection Control (ADRC) as a Control Tool for Practitioners. Electronics, 2013, 2(3),pp.246-279.

11. Ti. TMS320F28069M. TMS320F28068M InstaSPIN(TM) MOTION Software Technical Reference Manual: TEXAS INSTRUMENTS, 2013.

12. Shaughnessy J D, Pinckney S Z, McMinn J D, Hypersonic vehicle simulation model: winged-cone configuration. NASA TM-102610,1990.

13. Huang Yi,Han Jing-qing.Analysis and design of nonlinear two-order continuous extended state observer.Chinese Science Bulletin, 2000,45(13), pp. 1373-1379. 